\title{
ASSOCIATION OF THE EXPRESSION LEVEL OF THE MYBPC1 GENE IN SKELETAL MUSCLE WITH MARBLING TRAIT IN JAPANESE BLACK BEEF CATTLE*
}

\author{
Bin Tong $^{1 \star}$, Youji Muramatsu ${ }^{2}$, Takeshi Ohta ${ }^{3}$, Hiroyuki Kose ${ }^{4}$, Hideaki Yamashiro $^{1}$, Toshie Sugiyama ${ }^{1}$, \\ Takashisa Yamada ${ }^{1}$
}

${ }^{1}$ Faculty of Agriculture, Department of Agrobiology, Niigata University, 950-2181 Niigata, Japan ${ }^{2}$ Faculty of Health Sciences for Welfare, Department of Nutritional Sciences for Well-being, Kansai University of Welfare Sciences, 582-0026 Osaka, Japan

${ }^{3}$ Central Pharmaceutical Research Institute, Japan Tobacco, Inc., 569-1125 Osaka, Japan

${ }^{4}$ Department of Life Science, Division of Natural Sciences, International Christian University, 181-8585 Tokyo, Japan

•Corresponding author: tongbin87@gmail.com

\begin{abstract}
Marbling characterized by the amount and distribution of intramuscular fat (IMF) in longissimus muscle (LM) and measured as beef marbling score (BMS), is an economically important trait of beef cattle in Japan. The myosin binding protein C, slow type (MYBPC1) gene, involved in efficient energy metabolism and homeostasis during muscle contraction in slow skeletal muscle, has been previously shown to be expressed at different levels in the LM between high-marbled and lowmarbled steer groups using differential-display PCR (ddPCR). In this study, we found that IMF area (\%) in the sacrococcygeus muscle (SM) was positively correlated with BMS in the LM in Japanese Black steers $(n=22, r=0.941, P<0.0001)$. This suggested that the IMF area $(\%)$ in the $S M$ tends to equate marbling level in the LM. Furthermore, we showed that the MYBPC1 expression level in SM was significantly higher in the Japanese Black steers $(n=5)$ with high BMS than in the Japanese Black steers $(n=5)$ with low BMS $(\mathbf{P}<0.001)$. Moreover, correlation analyses showed that the expression level of the $M Y B P C 1$ gene was positively correlated with IMF area (\%) $(n=22$, $\mathrm{r}=0.858, \mathrm{P}<0.0001)$ and $\mathrm{BMS}(\mathrm{n}=22, \mathrm{r}=0.769, \mathrm{P}<0.0001)$, indicating the association of $M Y B P C 1$ expression level with marbling trait. These results, together with the previous ddPCR result, suggested that high level of $M Y B P C 1$ expression may be associated with the development of marbling in Japanese Black beef cattle.
\end{abstract}

Key words: intramuscular fat, Japanese Black breed, marbling, MYBPC1, skeletal muscle

Marbling is characterized as the amount and distribution of intramuscular fat (IMF) in a cross section of longissimus muscle (LM) in Japan. High levels of mar-

\footnotetext{
*This work was supported by a Grant-in-Aid for Scientific Research (B) (no. 14360166) from the Ministry of Education, Culture, Sports, Science and Technology of Japan, and by the research funds of Japanese Livestock Technology Association.
} 
bling improve the palatability and acceptability of the meat (Busboom et al., 1993; Boylston et al., 1995; Matsuishi et al., 2001). Because of the importance of marbling for the economics of beef production, it is greatly interesting to obtain better knowledge on the molecular architecture of marbling.

Marbling involves a series of events that initiate and maintain preadipocyte proliferation, differentiation of preadipocytes into adipocytes and adipocyte maturation throughout LM (Smith et al., 2000). The physiological or anatomical environment surrounding adipocyte-lineage cells, as well as intramuscular adipocyte-lineage cells are thought to contribute to specific adipogenic events involved in marbling (Smith et al., 2000). The environment can promote proliferation, differentiation or maturation of adipocyte-lineage cells throughout the muscle by many mechanisms including controlling energy balance, controlling the structural integrity of the sarcomere and affecting intramuscular vascularization. Recently, several studies focused on the expression of adipogenic and lipogenic-related genes in LM. For example, the expression levels of ADIPOQ, SCD and THRSP genes (Wang et al., 2009), and GPATl gene (Jeong et al., 2012) exhibited significant positive correlations with IMF content in LM. So far, a well-known biochemical finding in relation to marbling expression in muscle was presented by Jurie et al. (2007), who reported that FABP4 activity was strongly correlated with IMF content. However, studies about expression of energy metabolism-related gene in the environment surrounding IMF are limited.

From the result of our previous differential-display PCR (ddPCR) analysis in LM, the C25-36\#2 expressed sequence tag (EST) showed higher expression levels in high-marbled steer group than in low-marbled steer group (Sasaki et al., 2006). The C25-36\#2 EST sequence corresponds to a portion of the myosin binding protein $C$, slow type (MYBPC1) gene. MYBPC1 is known to be one isoform (in slow skeletal muscle) of myosin binding protein C (Offer et al., 1973; Pepe and Drucker, 1975; Sato et al., 2003). MYBPC1 acts as an adaptor to connect the ATP consumer (myosin) and the regenerator (MM-CK) for efficient energy metabolism and homoeostasis (Chen et al., 2011). The differential expression suggested that the increase in the $M Y B P C 1$ expression level might promote proliferation, differentiation, or maturation of adipocyte-lineage cells by promoting transduction and storage of energy in LM, thereby resulting in high levels of marbling. Thus, the MYBPC1 gene has been regarded as a functional candidate for the gene responsible for marbling. In this regard, it is important to understand the relationship between $M Y B P C 1$ expression level and marbling trait in a wide range of marbling scores.

In addition, Brackebusch et al., (1991) found that LM fat content was positively related to fat content of other 14 major muscles, especially related to marbling score. Furthermore, Osawa et al., (2008) reported that fat area ratio in the trapezius muscle was positively correlated with that in the LM and marbling score in Japanese Black beef cattle. These studies indicated that the IMF area ratio in the other muscles, such as sacrococcygeus muscle (SM), might be correlated with marbling score in the LM.

In this study, we investigated association between $M Y B P C 1$ expression level and marbling trait using the SM in Japanese Black beef cattle. 


\section{Material and methods}

\section{Samples and data}

We sampled SM tissues of 60 Japanese Black steers from a slaughterhouse at Niigata prefecture (Niigata, Japan). These steers were reared on the same diets for fattening period between 28 and 30 months, using commercial standard procedures of the Niigata Prefectural Headquarters, National Federation of Agricultural Cooperative Association (JA, Niigata, Japan). After slaughter, the SM tissues were removed from the root of tail, and trimmed of any external fat. For histology, the muscle tissues $(1 \times 1 \times 1 \mathrm{~cm})$ were taken from the muscle samples. For RNA extraction, $50 \mathrm{mg}$ muscle samples were taken, rapidly frozen in liquid nitrogen and stored at $-80^{\circ} \mathrm{C}$. After we obtained beef marbling standard number (BMS) data of each steer, the SM samples of 22 steers ( 1 to 2 steers per sire) from 18 sires were selected to represent a wide range of marbling scores (BMS 2 to 12). There was no strong bias for a specific father or a specific maternal grandfather of the sires, showing no genetic relationship in the 22 Japanese Black steers.

Marbling was measured by certified graders from the Japan Meat Grading Association (Tokyo, Japan), according to the Japanese meat grading system on carcasses dissected at the 6th and 7th rib section (JMGA, 1988). Marbling was scored from 1 to 12 BMS with a standard model panel, in which higher scores correspond to more IMF. The BMS date of each steer was obtained from JA (Niigata, Japan). This study conformed to the guidelines for animal experimentation of the Graduate School of Science and Technology, Niigata University (Niigata, Japan).

\section{Histology}

For each sample $(n=22), 5$ serial coronal sections $(15 \mu$ m thick $)$ were obtained at intervals of $200 \mu \mathrm{m}$ from the SM samples, and the sections stained with Oil Red $\mathrm{O}$ according to the protocol reported by Annika et al., (2013), to evaluate IMF area. Histology images were captured with an Eclipse microscope (model E400; Nikon, Tokyo, Japan) equipped with a high-resolution digital camera (DS-Ri1, Nikon) connected to a personal computer equipped with NIS-Elements software (Nikon) for image capture and archiving. Within each section, three nonoverlapping areas were randomly selected and digitally captured (20× magnification). In each of the digitally captured images, IMF area positive for Oil Red $\mathrm{O}$ staining was manually outlined and used to calculate the total area of IMF that was then divided by the total area of the image $\left(13.4 \mathrm{~mm}^{2}\right)$ to calculate the percent IMF area. The average percent IMF area of three images for each SM section was then determined. The average percentage of IMF area among replicate SM sections for each animal was calculated for subsequent use in correlation analysis.

\section{RNA extraction and real-time PCR}

For each steer ( $\mathrm{n}=22$ ), total RNA was isolated from $50 \mathrm{mg}$ of frozen SM samples using the RNeasy Fibrous Tissue kit (QIAGEN GmbH, Hilden, Germany) according to the manufacturer's instructions. Total RNA was quantified by absorbance at $260 \mathrm{~nm}$, and the integrity of total RNA was checked by agarose gel 
electrophoresis and ethidium bromide staining of the $28 \mathrm{~S}$ and $18 \mathrm{~S}$ bands. Total RNA $(2 \mu \mathrm{g})$ was reverse-transcribed into cDNA using an iScript Advanced cDNA Synthesis kit for RT-qPCR (Bio-Rad Laboratories, Hercules, CA, USA), according to the manufacturer's instructions. Real-time PCR was performed using SsoAdvanced SYBR Green Supermix (Bio-Rad). MYBPC1 mRNA expression level in the SM samples was determined with the MiniOpticon Real-Time PCR Detection System (Bio-Rad), using the MYBPC1 mRNA-specific primers (F: 5'CTCCTACTCTTCTGACCGTT-3' and R:5'-CACATAGATCCTTGAATCCGTT-3'). $G A P D H$ transcripts were amplified for normalization within each sample, using the GAPDH mRNA-specific primers (F: 5'-TGACCCCTTCATTGACCTTCA-3' and R: 5'-ACCCCAGTGGACTCCACCACAT-3'). The reaction was performed in $20 \mu \mathrm{l}$, containing $10 \mu \mathrm{l}$ SsoAdvanced SYBR Green Supermix (Bio-Rad), $1 \mu$ l of each primer $(10 \mu \mathrm{M}), 2 \mu \mathrm{l}$ cDNA $(2.5 \mathrm{ng} / \mu \mathrm{l})$ and $6 \mu \mathrm{l}$ Rase/DNase-free $\mathrm{H}_{2} \mathrm{O}$. The thermal cycling parameters were as follows: $95^{\circ} \mathrm{C}$ for $30 \mathrm{~s}$, followed by 40 cycles at $95^{\circ} \mathrm{C}$ for $3 \mathrm{~s}$ and $60^{\circ} \mathrm{C}$ for $30 \mathrm{~s}$. The relative fold change was calculated using the $2^{-\Delta \Delta \mathrm{Ct}}$ calculation (Schmittgen and Livak, 2008). The mean expression levels were obtained from three independent experiments.

\section{Data analyses}

Correlation analyses $(\mathrm{n}=22)$ between IMF area $(\%)$ and BMS, between MYBPC1 expression level and IMF area (\%), and between BMS and MYBPC1 expression level were calculated using Pearson's correlation coefficient (r). Comparison of MYBPC1 expression level in SM between 2 Japanese Black steer groups (5 steers with the highest BMS and 5 steers with the lowest BMS) were performed by Student's t test. P-values less than 0.05 were considered to be statistically significant.

\section{Results}

The coronal sections of the SM were stained with Oil Red O. The section area positive for Oil Red $\mathrm{O}$ staining was visually larger in high-marbled JB steer $(\mathrm{BMS}=11, \mathrm{IMF}$ area $=37.8 \%)$ than in low-marbled one $(\mathrm{BMS}=3, \mathrm{IMF}$ area $=7.0 \%)$ (Figure 1). The IMF area (\%) in the SM was positively correlated with BMS in the LM ( $\mathrm{n}=22, \mathrm{r}=0.941, \mathrm{P}<0.0001)$ (Figure 2). The increase in the IMF area $(\%)$ in the $\mathrm{SM}$ was correlated with the increase in BMS in the LM, suggesting that the IMF area $(\%)$ in the SM tends to equate marbling level in the LM.

Our previous ddPCR result showed that the expression level of the MYBPC1 gene in the LM was higher in high-marbled steer group than in low-marbled steer group (Sasaki et al., 2006). To replicate this result in the SM, we selected 10 SM samples of unrelated steers with the highest and the lowest BMS. The average BMS of the 5 steers with the highest BMS or the lowest BMS was 3 (ranging from 2 to 4) or 11 (ranging from 10 to 12), respectively. The expression level of the MYBPC1 gene was significantly higher in the steers with the highest BMS than in the steers 
with the lowest BMS $(\mathrm{P}<0.001)$ (Figure 3). This result suggests the MYBPC1 gene has similar expression pattern between SM and LM

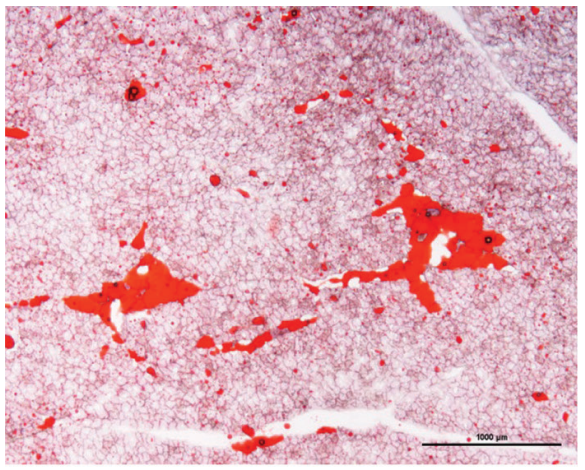

BMS 3

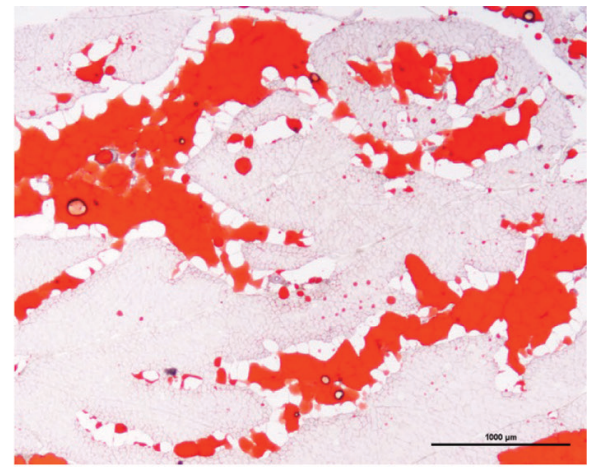

BMS 11

Figure 1. Oil Red O staining of the coronal sections of SM from steers with marbling score (BMS) 3 and BMS 11. The area positive for Oil Red O staining is shown by red color

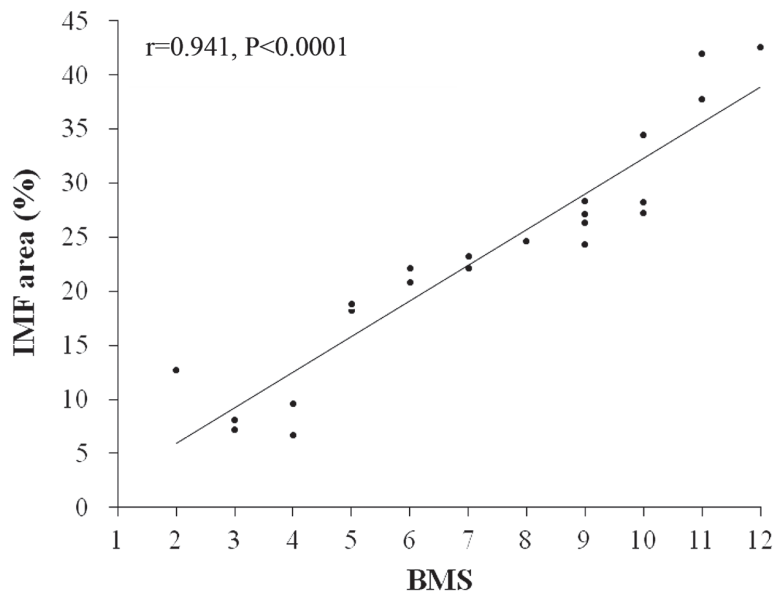

Figure 2. Correlation between beef marbling score (BMS) and intramuscular fat (IMF) area (\%) in the $\mathrm{SM}$ of the Japanese Black steers $(\mathrm{n}=22)$. The corresponding r value represents the Pearson's correlation coefficient

The correlation between the MYBPC1 expression level and the percentage of IMF area is presented in Figure 4. The percentages of IMF area in the SM represent a wide range from 5.7 to $42.5 \%$ (Figure 2). The expression level of the $M Y B P C 1$ gene was positively correlated with IMF area (\%) in the $\mathrm{SM}(\mathrm{n}=22, \mathrm{r}=0.858, \mathrm{P}<0.0001)$ (Figure 4). Furthermore, the expression level of the MYBPC1 gene was positively correlated with BMS $(\mathrm{n}=22, \mathrm{r}=0.769, \mathrm{P}<0.0001)$ (Figure 5). These results suggest the $M Y B P C 1$ expression level is associated with marbling trait. 


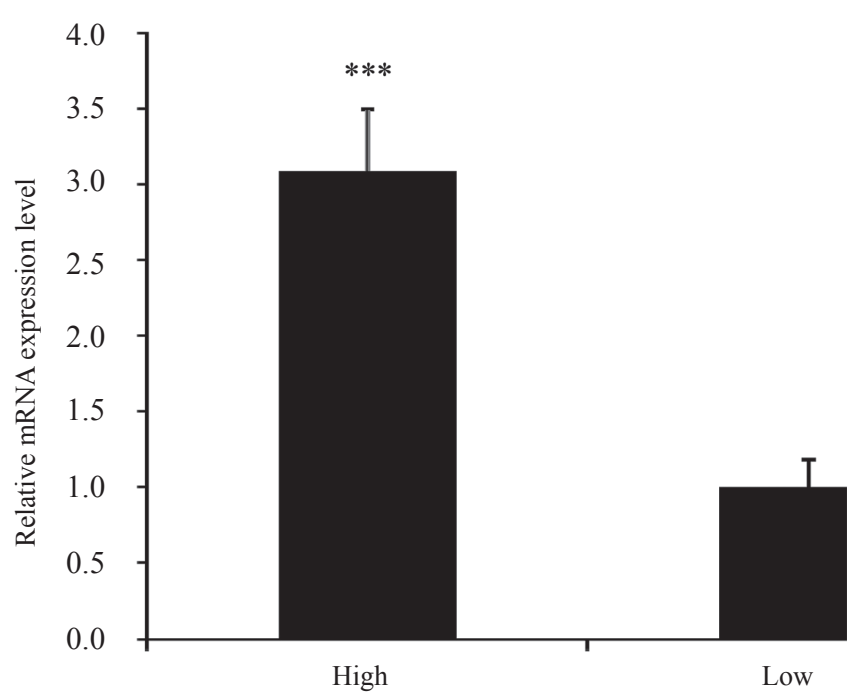

Figure 3. Expression levels of the $M Y B P C 1$ gene in the SM of 2 Japanese Black steer groups with the highest BMS and the lowest one. High, 5 steers with the highest BMS. Low, 5 steers with the lowest BMS. Expression levels were determined by real-time PCR and normalized to GAPDH. Expression levels of 5 steers with the lowest BMS were normalized to 1.0. Values are mean \pm SEM. Significant difference: $* * * \mathrm{P}<0.001$

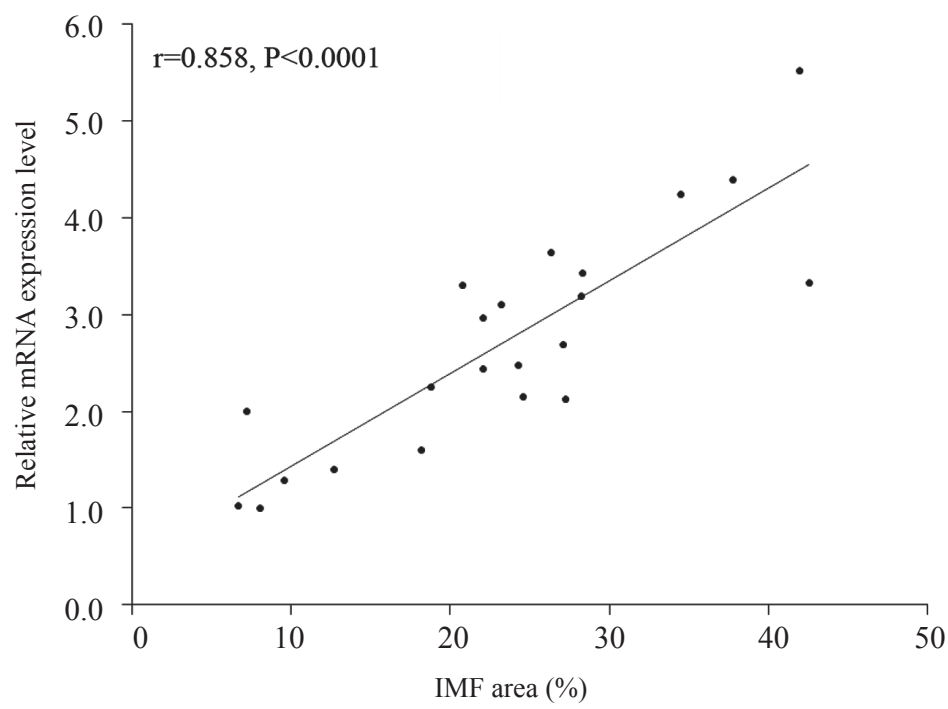

Figure 4. Correlation between $M Y B P C 1$ expression level and intramuscular fat (IMF) area (\%) in the $\mathrm{SM}$ of the Japanese Black steers $(\mathrm{n}=22)$. The corresponding r value represents the Pearson's correlation coefficient 


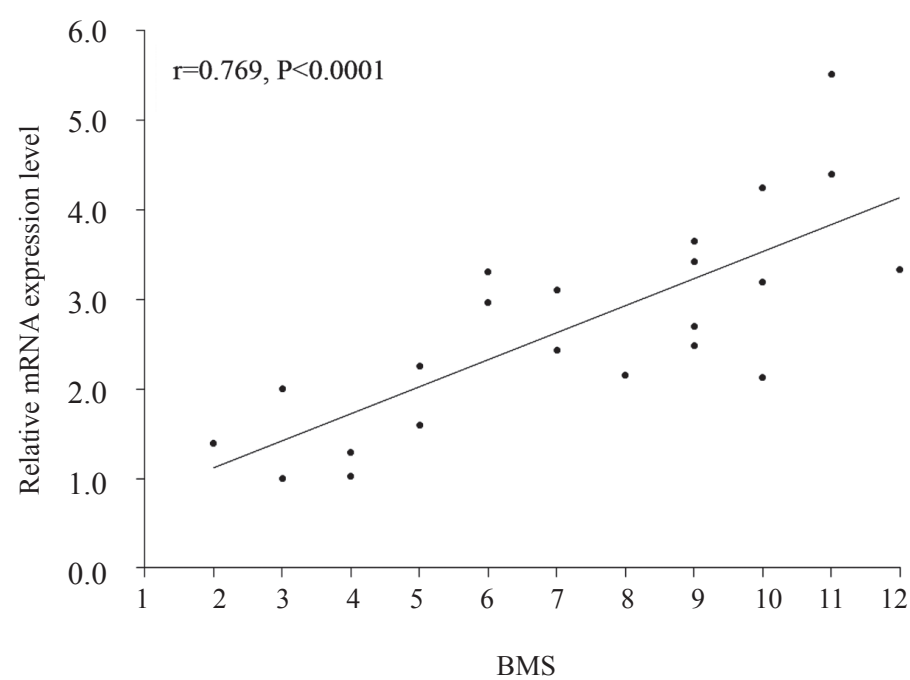

Figure 5. Correlation between $M Y B P C 1$ expression level and beef marbling score (BMS) in the SM of the Japanese Black steers $(n=22)$. The corresponding r value represents the Pearson's correlation coefficient

\section{Discussion}

Marbling (intramuscular fat) accumulates in a connective tissue matrix in close proximity to a blood capillary network between the bundle of muscle fibers in bovine skeletal muscle (Harper and Pethick, 2004). The physiological or anatomical environment can promote proliferation, differentiation or maturation of adipocyte-lineage cells throughout the muscle by many mechanisms including controlling energy balance. We have previously undertaken ddPCR in low-marbled and high-marbled steer groups to explore genes showing marbling-associated expression changes in LM (Sasaki et al., 2006). Among the detected genes, the EDG1 gene, which is known to be involved in blood vessel formation (Liu et al., 2000), showed higher expression levels in high-marbled steer group than in low-marbled steer group. The increase of EDG1 expression may promote proliferation, differentiation or maturation of adipocyte-lineage cells by promoting intramuscular vascularization and then energy provision for the muscle, thereby resulting in high levels of marbling (Yamada et al., 2009). From the result of ddPCR analysis, the MYBPC1 gene (C25-36\#2 EST) showed higher expression levels in high-marbled steer group than in low-marbled steer group. MYBPC1 is known to encode the slow skeletal muscle isoform of the major myosin-binding proteins in vertebrate striated muscles (Offer et al., 1973; Pepe and Drucker, 1975; Sato et al., 2003) and act as an adaptor to connect the ATP consumer (myosin) and the regenerator (MM-CK) for efficient energy metabolism and homoeostasis (Chen et al., 2011). It has been considered as a functional candidate gene for marbling trait. In this study, the correlation analysis showed that the 
MYBPC1 expression level was positively correlated with IMF area (\%). Furthermore, the expression level of the MYBPC1 gene was significantly higher in the steers with the highest BMS than in the steers with the lowest BMS. These results indicated that the higher expression level of the MYBPC1 gene could result in more IMF deposition in the skeletal muscle by controlling the energy metabolism and homoeostasis of slow skeletal muscle.

In addition, marbling (intramuscular fat) is a major trait in characterizing beef quality and an important factor for determining the price of beef in Japan (JMGA, 1988). Especially in Japan, the LM tissue is not conveniently available from the commercially slaughtered carcass market because of high economic value of this muscle, but the SM is. To date, many previous researches have indicated that marbling in the LM has a positive correlation with fat deposition in the other muscles using different meat quality measurements (Garrett and Hinman, 1971; Brackebusch et al., 1991; Park et al., 1994; Yang et al., 2006; Osawa et al., 2008). In agreement with previous studies, the strongly positive correlation between the IMF area (\%) in SM and BMS in LM was obtained in this study. Moreover, the MYBPC1 gene has similar expression pattern between SM and LM, so we hypothesize that the expression profiles of the marbling-related genes in SM are similar to those in LM. Therefore, for future study, we have to investigate the expression level of the other marbling-related genes in the SM, such as EDG1, TTN and SORBS1 gene detected in our previous ddPCR (Sasaki et al., 2006).

Based on the positive correlation between $M Y B P C 1$ expression level and IMF area (\%) in the SM, together with the similar expression pattern of $M Y B P C 1$ between the LM and the SM, we suggested the expression level of $M Y B P C 1$ is associated the marbling trait. We have recently reported that the $\mathrm{G}$ allele at the g.70014208A $>\mathrm{G}$ SNP in the promoter region of the MYBPCl gene was associated with high marbling level in Japanese Black beef cattle (Tong et al., 2014). These results showed that $M Y B P C 1$ is one of major functional genes that is associated with marbling trait. We also hypothesized that the g.70014208A $>$ G SNP might have an impact on $M Y B P C 1$ expression and also marbling by affecting $M Y B P C 1$ promoter activity. Further study will be needed to clarify effect of the SNP on the MYBPC1 expression level.

\section{References}

A nnika M., C a rolina E.H., Lars M., Ulf E., Anneli e F. (2013). Imaging of neutral lipids by oil red $\mathrm{O}$ for analyzing the metabolic status in health and disease. Nat. Protoc., 8: 1149-1154.

B o yl s t on T.D., M organ S.A., J o hn s o n K.A., B u s b o o m J.R., Wright R.W., R e e ve s J.J. (1995). Lipid-content and composition of Wagyu and domestic breeds of beef. J. Agric. Food Chem., 43: $1202-1207$.

B ra c k e bu s ch S.A., M c K e i th F.K., C a r r T.R., M c L are n D.G. (1991). Relationship between longissimus composition and the composition of other major muscles of the beef carcass. J. Anim. Sci., 69: 631-640. 
B u s b o o m J.R., J e r e m i a h L.E., G ib s o n L.L., J o hn s o n K.A., G a s k in s C.T., R e e v e s J.J., Wright R.W. (1993). Effects of biological source on cooking and palatability attributes of beef produced for the Japanese market. Meat Sci., 35: 241-258.

Chen Z., Zh a o T.J., L i J., G a o Y.S., Meng F.G., Yan Y.B., Z hou H.M. (2011). Slow skeletal muscle myosin-binding protein-C $(M Y B P C 1)$ mediates recruitment of muscle-type creatine kinase (CK) to myosin. Biochem. J., 436: 437-445.

Garret t W.N., H in ma n N. (1971). Fat content of trimmed beef muscles as influenced by quality grade, yield grade, marbling score and sex. J. Anim. Sci., 33: 948-957.

Harper G.S., Pethick D.W. (2004). How might marbling begin? Aust. J. Exp. Agric., 44: $653-662$.

J e o n g J., K w o n E.G., I m S.K., S e o K.S., B a i k M. (2012). Expression of fat deposition and fat removal genes is associated with intramuscular fat content in longissimus dorsi muscle of Korean cattle steers. J. Anim. Sci., 90: 2044-2053.

JMGA (1988). New Beef Carcass Grading Standards. Japan Meat Grading Association, Tokyo, Japan.

Jurie C., Cassar-Malek I., Bonnet M., Leroux C., Bauchart D., Boulesteix P., Pethick D.W., Hocquette J.F. (2007). Adipocyte fatty acid-binding protein and mitochondrial enzyme activities in muscles as relevant indicators of marbling in cattle. J. Anim. Sci., 85: 2660-2669.

Liu Y., Wada R., Yamashita T., Mi Y., Deng C.X., Hobson J.P., Rosenfeldt H.M., Nava V.E., Chae S.S., Lee M.J., Liu C.H., Hla T., Spiegel S., Proia R.L. (2000). Edg-1, the G protein-coupled receptor for sphingosine-1-phosphate, is essential for vascular maturation. J. Clin. Invest., 106: 951-961.

Mat s u is hi M., F u j i m or i M., O k i t a n i A. (2001). Wagyu beef aroma in Wagyu (Japanese Black cattle) beef preferred by the Japanese over imported beef. Anim. Sci. J., 72: 498-504.

O ffer G., Moos C., S t a r r R. (1973). A new protein of the thick filaments of vertebrate skeletal myofibrils: Extraction, purification and characterization. J. Mol. Biol., 74: 653-676.

Os aw a T., Kuchida K., Hidaka S., Kato T. (2008). Genetic parameters for image analysis traits on M. longissimus thoracis and M. trapezius of carcass cross section in Japanese Black cattle. J. Anim. Sci., 86: 40-46.

P a rk B., Whittaker A.D., M ille r R.K., H a le D.S. (1994). Predicting intramuscular fat in beef longissimus muscle from speed of sound. J. Anim. Sci., 72: 109-116.

P e pe F.A., Dru c k e r B. (1975). The myosin filament: III. C-protein. J. Mol. Biol., 99: 609-617.

S a saki Y., Nagai K., Nagat a Y., Doronbekov K., Nishimura S., Yoshioka S., Fujita T., Shiga K., Miyake T., Taniguchi Y., Yamada T. (2006). Exploration of genes showing intramuscular fat deposition-associated expression changes in longissimus muscle. Anim. Genet., 37: 40-46.

Sato N., Kawakami T., Nakayama A., Suzuki H., Kasahara H., Obinata T. (2003). A novel variant of cardiac myosin-binding protein-C that is unable to assemble into sarcomeres is expressed in the aged mouse atrium. Mol. Biol. Cell., 14: 3180-3191.

S chmittgen T.D., Livak K.J. (2008). Analyzing real-time PCR data by the comparative C (T) method. Nat. Protoc., 3: 1101-1108.

S m it h S.B., L u n t D.K., Z e m b a y a s h i M. (2000). Intramuscular fat deposition: The physiological process and the potential for its manipulation. Plains Nutritional Council Spring Conference, Texas A\& M Research and Extension Centre, San Antonio Texas, USA, pp. 1-12.

Tong B., Sasaki S., Muramatsu Y., Ohta T., Kose H., Yamashiro H., Fujita T., Yamada T. (2014). Association of a single nucleotide polymorphism in myosin binding protein $\mathrm{C}$, slow type $(M Y B P C 1)$ gene with marbling in Japanese Black beef cattle. Anim. Genet., 45 : $611-612$.

W a g Y.H., B o w e r N.I., R e v e rt e r A., Tan S.H., D e J a ge r N., W a n g R., M c Wi 11 i a m S.M., C a fe L.M., Gre e n w o o d P.L., L e hn e rt S.A. (2009). Gene expression patterns during intramuscular fat development in cattle. J. Anim. Sci., 87: 119-130.

Yamada T., Itoh M., Nishimura S., Taniguchi Y., Miyake T., Sasaki S., Yoshiok a S., F ujit a T., S hig a K., M orit a M., S a s a ki Y. (2009). Association of single nucleotide polymorphisms in endothelial differentiation, sphingolipid G-protein-coupled receptor, 1 gene with marbling in Japanese Black beef cattle. Anim. Genet., 40: 209-216. 
Yang X.J., A lbrecht E., End er K., Zh a o R.Q., We gne r J. (2006). Computer image analysis of intramuscular adipocytes and marbling in the longissimus muscle of cattle. J. Anim. Sci., 84: $3251-3258$.

Received: 5 X 2014

Accepted: 5 I 2015 